NBER WORKING PAPER SERIES

\title{
PRIMARY CARE PHYSICIAN PRACTICE STYLES AND PATIENT CARE: EVIDENCE FROM PHYSICIAN EXITS IN MEDICARE
}

\author{
Itzik Fadlon \\ Jessica N. Van Parys \\ Working Paper 26269 \\ http://www.nber.org/papers/w26269 \\ NATIONAL BUREAU OF ECONOMIC RESEARCH \\ 1050 Massachusetts Avenue \\ Cambridge, MA 02138 \\ September 2019
}

We thank Leila Agha, Jeff Clemens, Julie Cullen, Amy Finkelstein, Jon Skinner, and Nicolas Ziebarth for their helpful comments on the paper. We also thank the participants at the following seminars and conferences: The Dartmouth Institute P01 meetings in April 2017 and 2018, the 2018 ASHEcon Conference, the Fall 2018 CUNY GC and RPI Seminars, the 2019 BU-HarvardMIT Health Seminar, and the 2019 NBER-CEPRA Conference. Research reported in this paper was supported by the National Institute on Aging of the National Institutes of Health under Award Number P01AG005842. The content is solely the responsibility of the authors and does not necessarily represent the official views of the National Institutes of Health or the National Bureau of Economic Research.

NBER working papers are circulated for discussion and comment purposes. They have not been peer-reviewed or been subject to the review by the NBER Board of Directors that accompanies official NBER publications.

(C) 2019 by Itzik Fadlon and Jessica N. Van Parys. All rights reserved. Short sections of text, not to exceed two paragraphs, may be quoted without explicit permission provided that full credit, including $(\odot$ notice, is given to the source. 
Primary Care Physician Practice Styles and Patient Care: Evidence from Physician Exits in Medicare Itzik Fadlon and Jessica N. Van Parys

NBER Working Paper No. 26269

September 2019

JEL No. I11,I13,I18

\begin{abstract}
Primary care physicians (PCPs) provide frontline health care to patients in the U.S.; however, it is unclear how their practice styles affect patient care. In this paper, we estimate the long-lasting effects of PCP practice styles on patient health care utilization by focusing on Medicare patients affected by PCP relocations or retirements, which we refer to as "exits." Observing where patients receive care after these exits, we estimate event studies to compare patients who switch to PCPs with different practice style intensities. We find that PCPs have large effects on a range of aggregate utilization measures, including physician and outpatient spending and the number of diagnosed conditions. Moreover, we find that PCPs have large effects on the quality of care that patients receive, and that all of these effects persist for several years. Our results suggest that switching to higher-quality PCPs could significantly affect patients' longer-run health outcomes.
\end{abstract}

\author{
Itzik Fadlon \\ Department of Economics \\ University of California, San Diego \\ 9500 Gilman Drive, \#0508 \\ La Jolla, CA 92093 \\ and NBER \\ fadlon@ucsd.edu \\ Jessica N. Van Parys \\ Department of Economics \\ Hunter College \\ 695 Park Avenue, HW 1534 \\ New York, NY 10065 \\ jessica.vanparys@hunter.cuny.edu
}




\section{Introduction}

Medicare spending comprises $\$ 706$ billion of the U.S. federal budget, and it is projected to grow by more than $7 \%$ over the next decade (CMS 2019). For reasons that have not been fully understood, Medicare spending has varied significantly across patients in the U.S., both within and across geographical regions (Fisher et al. 2003a,b; Sinaiko et al. 2019). Understanding what drives variation in utilization is important for policy efforts that aim to reduce spending and improve care. For example, if demand side factors, such as patients' medical needs, drive spending differences across patients, then policies that improve patient health may reduce health care spending. On the other hand, if supply side factors, such as physician beliefs or incentives, predominantly drive spending, then policies that target the provider side may prove more effective.

Accordingly, recent research has identified factors that drive the variation in health care spending and utilization across patients in the U.S. Specifically, Finkelstein et al. (2016) decompose regional spending variation into its demand and supply side components by analyzing Medicare patients who move across regions. They conclude that $40-50 \%$ of the cross-regional variation is due to supply side factors.$^{1}$ In addition, a growing body of research documents variation in utilization and quality of care across specific health care providers, ranging from hospitals to specialists, and examines the implications of such "practice style" variation for patient outcomes..$^{2}$

Within the practice style literature, however, primary care physicians (PCPs) are a relatively understudied provider group. Characterizing PCP practice styles and estimating their effects on patients' utilization has been hindered by data challenges-i.e., the limited availability of large-scale data sets that include patient-provider linkages - as well as concerns about patient-physician selection. Yet, PCPs could be responsible for significant variation in utilization and patient outcomes

\footnotetext{
${ }^{1}$ Song et al. (2010) use a similar approach and find that regional practice patterns also explain variation in the diagnoses that patients receive.

${ }^{2}$ For hospitals, see, e.g., Doyle (2011), Doyle et al. (2015), Hull (2018), and Doyle et al. (forthcoming). For specialists, see, e.g., Epstein and Nicholson (2009), Epstein et al. (2016) and Currie and MacLeod (2016) on obstetricians; Currie et al. (2016) and Molitor (2018) on cardiologists; Chan (2016), Van Parys (2016), Gowrisankaran et al. (2017), Gruber et al. (2018), and Silver (2019) on emergency department physicians; Fletcher et al. (2014) on hospital attending physicians; Sinaiko et al. (2019) on oncologists; and Tu (2017) and Agha et al. (2018) who use specialist exits to examine the role of physicians' referral networks.
} 
because they are institutionally positioned to play a central role in health care provision. In the U.S., they provide frontline health care to most of the insured population, and they do so through several functions; they diagnose conditions, treat illnesses, prescribe drugs, and refer patients to specialists. PCPs also have more continuous interactions with their patients than other types of health care providers, who may only interact with patients occasionally to treat acute conditions. The fact that PCPs are so intricately involved with health care provision means that PCP practice styles could significantly affect patients' health care utilization trajectories, particularly if patients lack information about the kind of care they should receive.

In this paper, we show how PCP practice style intensity-across a wide range of measures and functions - affects the care that patients receive. We use data on a 20\% sample of Medicare patients from 2007-2013 to exploit variation in PCP practice styles within patients over time. Specifically, we follow 195,000 patients who switch PCPs after having lost their original primary care providers due to "exits," i.e., their PCPs move to other regions or leave the Medicare program. We focus on patients affected by PCP exits because the exits are likely exogenous to the patients' underlying health trajectories. As such, our variation in PCP practice styles comes from differences between the new and the old PCPs' practice style intensities. We further leverage the richness of the data to categorize PCP practice styles across many dimensions, ranging from office-based spending to total spending to emergency department visits and the probability that patients receive guidelineconsistent care. This allows us to analyze outcomes that are closer to and further from the PCPs' direct locus of control. We are thus able to assess the range of impacts that PCPs have on patients' health care utilization, measured in terms of quantity and quality.

Our research design employs a standard event-study approach where the treatment intensity varies across patients who are affected by PCP exits. We estimate dynamic difference-in-differences specifications for each health care utilization outcome, where we show how utilization evolves around the PCP exit for patients with varying degrees of changes in practice style intensity between their new and old PCPs. Our stylized statistical model, which closely follows Finkelstein et al. (2016), shows that our event study estimates measure the share of variation in utilization across 
patients within regions that is attributable to PCP practice styles. The identifying assumption in our quasi-experiment is that the underlying trends in health care utilization across patients who experience differential changes in practice style intensity after their PCPs exit do not systematically differ. In support of this assumption, we show that there are virtually no differential pre-trends across patients with varying degrees of treatment intensity. In addition, we find little evidence (precisely estimated) that our results can be explained by patients sorting to new PCPs based on observables.

Our main findings are as follows. Across our entire set of outcomes, PCP practice styles immediately impact patient health care utilization at the time of a new patient-physician match. Further, the effects persist for at least six years after the new match. To varying degrees, PCP practice styles affect all types of spending (i.e., physician, drug, outpatient, inpatient, post-acute care, and total), encounters with different types of health care providers (i.e., numbers of office visits, emergency department visits, and avoidable hospitalizations), the numbers of diagnoses that patients receive, and the probability that patients receive guideline-consistent care (i.e., flu vaccinations; eye exams and $\mathrm{A} 1 \mathrm{C}$ and lipid screening tests for diabetic patients). As an example, we find that patients who switch to PCPs with $\$ 100$ higher physician-spending intensity see their own physician-related spending increase by an average of $\$ 54$ in the years following the switch. Importantly, the effects of PCP practice styles are even larger for outcomes related to high-quality care. When we examine influenza vaccinations, which are recommended annually for all patients over 65 years old, we find that patients who switch to PCPs with 10 percentage point higher vaccination rates are 6 percentage points more likely to get vaccinated themselves. The relationship between PCPs' propensity to provide guideline-consistent care for diabetic patients and patients' receipt of such care is even larger. Overall, our results point to far-reaching and long-lasting effects of primary care physician practice styles on both the quantity and quality of patient health care utilization and, hence, potentially on patient health. In turn, our results suggest that health policies aimed at expanding high-value care and reducing low-value care might be most effective if targeted at PCPs. 
Some recent and concurrent work studies primary care physicians and their practice styles in countries other than the U.S. In Denmark, Koulayev et al. (2017) study whether PCPs affect patients' adherence to medications; Laird and Nielsen (2018) use patient migrations to study regional variation in PCP prescribing rates; and Simonsen et al. (2019) use practice closures to examine the effects of health care disruption and assess the role of physician practice styles in explaining the disruption effects. In Norway, Grytten and Sorensen (2003) estimate variation across PCPs for a range of practice styles, and in Israel, Alkalay et al. (2018) examine how PCP practice patterns change in the face of time constraints. While these papers are indeed relevant and informative for our application, they study institutionally different settings; the U.S. health care system is known to be different from (and often an outlier to) other countries in the developed world in important aspects, including pricing, provider reimbursement, cost-effectiveness analysis, and performance $3^{3}$ There is arguably less regulation of medical care in the U.S., even in Traditional Medicare, and patients may have more choices over providers. Hence, variation in PCP practice styles and its effects on patient care may be different in the U.S. relative to other OECD countries.

Our paper is most closely related to the recent limited work on primary care physicians in the United States. Cutler et al. (2019) use vignettes from surveys of patients and physicians, linked to Medicare expenditures, to determine whether patient factors or physician factors (such as physician beliefs about treatment) explain regional variation in spending. In contemporaneous working papers, Zhang (2018) and Kwok (2019) study patient-physician separations to focus on the consequences of primary care disruption in the Medicare population.$^{4}$ Kwok (2019) additionally studies how health care utilization differs across patients who switch to high versus low spending PCPs, combining both physician-initiated and patient-initiated switches.

Our paper contributes to the U.S.-focused literature on practice style variation in several ways. First, our approach to estimating the causal effects of PCP practice styles isolates variation that

\footnotetext{
${ }^{3}$ See, e.g., Garber and Skinner (2008), Cutler and Ly (2011), Laugesen and Glied (2011), Sawyer and Gonzales (2017), Schneider and Squires (2017), Schneider et al. (2017), and Papanicolas et al. (2018).

${ }^{4}$ Reddy et al. (2015) study the effect of primary care provider turnover on patient care and the quality of ambulatory care using a nationwide sample of patients in the Veterans Health Administration (VHA). Other related research shows the effects of primary care access on patient outcomes—see, e.g., Bailey and Goodman-Bacon (2015) who use the rollout of the first Community Health Centers (CHCs).
} 
is generated by plausibly exogenous patient-physician separations (i.e., PCP exits). Second, we examine the broadest range of PCP practice styles, including styles that reflect low-quality care (number of emergency department visits and avoidable hospitalizations) and styles that reflect high-quality care (flu vaccinations and recommended diabetes care). We additionally examine the complementarity between office-based care and other types of health care utilization, showing that patients who switch to PCPs who provide more office-based care often experience increases in spending on other types of care. Lastly, our paper contributes to the larger literature on health care providers and their quality in the U.S., where PCPs have been distinctly understudied. To the best of our knowledge, our paper may be the most comprehensive evaluation on the importance of PCP practice styles in the U.S. to date, providing evidence in support of large and persistent effects on the quantity and quality of care that patients receive.

The rest of the paper proceeds as follows. Section 2 describes the data and sample construction

process. Section 3 describes the empirical method. Section 4 presents the results, and Section 5 concludes with a brief discussion.

\section{Data and Analysis Sample}

In this section, we describe our data, present our analysis sample and explain how it is constructed, and define our outcomes that capture quantity and quality of health care utilization.

Our baseline data set is comprised of a 20\% sample of Medicare enrollees from 2007-2013. From these data, we keep observations of enrollees ages 65 and older with at least one month of Traditional Medicare (TM) enrollment in a given year (about 42 million enrollee-year observations), and we drop observations associated with enrollees who moved across Hospital Referral Regions (HRRs) over the time period (which equals about 3 million enrollee-year observations). We drop patients who move across regions because we do not want to use variation in PCP practice styles induced by enrollee relocations, but rather we want to focus on variation induced by PCP exits as we believe that PCP exits are more exogenous to patients' health trajectories. 
Based on these data, we construct our analysis sample in two steps. First, we construct a sample of primary care physicians. Second, we identify PCPs who exit during the time frame 2007-2013, and we derive a panel data set of the patients whose PCPs exited; the panel data set of patients affected by PCP exits comprises our final analysis sample. We now turn to describe the details of the procedures taken in these two steps.

To construct the sample of primary care physicians, we use the Medicare Carrier data to attribute Medicare enrollees to their primary care providers each year. We begin by identifying claims for Evaluation and Management (E\&M) visits, which reflect billing for office visits.$^{5}$ Out of these claims, we isolate claims associated with physicians whose primary specialty is family practice, internal medicine, or general medicine, which together comprise the typical specialities of primary care. Then for each patient that appears in these claims, we assign the primary care provider in a given year to be the National Provider ID (NPI) of the modal billing physician (among the providers with a typical primary care speciality). This matches patients to physicians in about $85 \%$ of the annual E\&M claims. For the remaining $15 \%$ of patient-years with E\&M claims that cannot be matched to physicians in a typical primary care specialty, we define the PCP as the modal physician who bills for E\&M claims, regardless of specialty. Since TM does not require patients to visit physicians with traditional PCP specialties, some patients may opt to see specialists as their "primary providers," and we do not want to discard observations where this is the case ${ }^{6}$

This procedure defines an overall sample of physicians who we refer to as PCPs. Next, we use the Carrier data to identify PCPs who relocate or stop treating TM patients. For each PCP, we gather all of the carrier line items where the physician is the performing provider, and we identify his/her modal billing zip code each year. If the first two digits of the modal billing zip code change from one year to the next, we say that the PCP has "relocated.' 7 Then, we classify PCPs who appear at least once, and then never again, as "retirements." We use the term "exit" to refer to

\footnotetext{
${ }^{5}$ We identify E\&M claims using the BETOS codes on the Carrier claim line items.

${ }^{6}$ In our final analysis sample, the average patient receives $64 \%$ of her E\&M care from the attributed PCP.

${ }^{7}$ The first two digits of a zip code identify state boundaries when states are small, and they identify large regions within states when states are large. The first two digits of zip codes cover larger geographic regions than hospital referral regions; there are 306 hospital referral regions in the U.S., but only 99 unique first two-digit zip codes.
} 
PCPs who either relocate or retire. Among the approximately 381,000 PCPs in the data, 26,587 relocate and 21,690 retire from the TM program at some point between 2007-2013. Finally, we identify all of the patients who matched to the exiting PCPs at some point in the pre-exit periods, and we drop patients who switched PCPs prior to an exit. This restriction guarantees that patients were exposed to one type of practice style in the pre-exit period. We call our final analysis sample the sample of patients affected by PCP exits; it includes 195,023 patients and 1,186,752 patientyear observations.

As a descriptive exercise, Tables 1 and 2 compare patients in our final analysis sample (in column 1) to patients who matched to PCPs, but who were not affected by exits (in column 2). While our empirical approach does not utilize such a comparison, it is nevertheless useful to show that the sample of patients affected by exits is quite similar to the sample of patients who were never affected. This provides suggestive evidence that PCPs relocate and retire for reasons that are unrelated to their patients' characteristics. The similarity between the two samples also speaks to the external validity of our results; that is, whether our results might apply to the broader Medicare population assignable to PCPs.

Turning to the description of our analysis sample, Table 1 first shows their average demographic characteristics and most common chronic conditions. The average patient is 76 years old, $59 \%$ of patients are female, and $14 \%$ are nonwhite. The vast majority have 12 months of Part $\mathrm{A}$ and B coverage, and the average number of HMO months is only 0.60 (given our use of the Carrier E\&M claims to define the sample). Approximately half of the sample has Part D coverage and $20 \%$ has the Part D low-income subsidy coverage. About $14 \%$ are dually enrolled in Medicare and Medicaid. Only 1\% qualifies for Medicare through disability or end-stage renal disease (ESRD) status. Among the 60 chronic and disabling conditions included in the Medicare Master Beneficiary Summary File (MBSF), the most common conditions are hypertension (63\%), hyperlipidemia/high cholesterol (51\%), ischemic heart disease (32\%), rheumatoid arthritis (30\%), diabetes (26\%), anemia (24\%), and cataracts (23\%).

The two upper panels of Table 2 show average health care utilization, including spending, 
numbers of visits, and numbers of diagnoses for patients in our sample. Average yearly total spending is $\$ 11,634$ per patient, and inpatient spending comprises the highest share of the total at $40 \%(\$ 4,654)$. Outpatient spending comprises $22 \%(\$ 2,551)$, pharmaceutical drug spending comprises $16 \%(\$ 1,877)$, and post-acute care spending comprises $16 \%(\$ 1,841)$ of total spending. Physician office-based spending makes up a much smaller share of the total at $5.2 \%(\$ 615)$ per year, and patients make an average of 8.1 office visits per year. Since physician spending per patient is so much less than inpatient and outpatient spending, this stresses the importance of characterizing PCP practice styles along a variety of dimensions. For example, PCPs' propensity to refer patients to inpatient and outpatient care might have more economically significant effects on total utilization than office-based care provided directly by the PCP. Finally, the average patient has 13.4 unique ICD-9 code diagnoses from all of their office visits each year, which amounts to 3.7 unique chronic conditions as measured on the Medicare MBSF Chronic Conditions Segment.

To identify health care utilization that can potentially reflect "low-quality" primary care, we focus on emergency department visits and hospitalizations for ambulatory care sensitive conditions (ACSCs), i.e., avoidable hospitalizations (using the 2017 CMS ACSCs). The idea is that patients who receive high-quality primary care should not visit the emergency department often and should not become hospitalized for avoidable reasons. For example, the Center for Medicare and Medicaid Services states that high rates of hospitalizations for ACSCs could indicate that "beneficiaries are not receiving high-quality ambulatory care," and that low rates of ACSCs at the provider level "may signify that the provider is providing better primary care and coordinating effectively with providers in the continuum of care" (CMS 2017). We find that about one in two enrollees has an emergency department visit each year and one in twenty enrollees has an avoidable hospitalization each year (see Table 2).

To identify health care utilization that can potentially reflect "high-quality" care, we focus on flu vaccinations for all Medicare patients, and guideline-consistent care for diabetic patients. First, since people 65 years and older are at high risk of serious complications from the flu (as the immune system becomes weaker with age), the guidelines recommend seasonal flu vaccinations each year 
for all patients in our elderly Medicare population (CDC 2019a,b). Moreover, Medicare Part B covers flu vaccinations and PCPs can easily administer the vaccines in office settings. We estimate that $45 \%$ of our sample receives flu vaccinations each year ${ }^{8}$ Second, clinical practice guidelines recommend that diabetic patients receive annual A1C tests, retina exams, and blood lipids tests to monitor their conditions (Goodman et al. 2010). We estimate that $46 \%$ of the diabetic patients in our sample receive all three tests each year (see Table 2 for these means). The advantages to using these practice styles as proxies for high-quality care are that (1) they are supported by medical research, and (2) we are able to study the relevant populations for whom the guidelines apply.

\section{Empirical Framework}

In this section, we describe our research design for estimating the effects of PCP practice styles on patients' health care utilization. We begin by describing a stylized statistical model of health care utilization, which follows Finkelstein et al. (2016) as applied to our setting, and we then describe the estimating equations that the model motivates. The statistical model provides a clear interpretation of our estimating equation's coefficients.

\subsection{Statistical Model}

Assume the following simple model for patient health care utilization: ${ }^{9}$

$$
y_{i d t}=\alpha_{i}+\delta_{d(i, t)}+x_{i t} \beta+\varepsilon_{i t}
$$

In this specification, $y_{i d t}$ is the value of outcome $y$ for patient $i$, who is treated by physician $d$ in year t. $\alpha_{i}$ is a patient fixed effect, and $\delta_{d(i, t)}$ is the effect of physician $d$ 's practice style (with regards

\footnotetext{
${ }^{8}$ We use the following HCPCS codes to identify patients who either received the flu vaccine or who were screened and found to be ineligible to receive the vaccine: G0008, G0919, G8108, G8110, G8423, G8426, G8482, G8636, G8637, G8639, G8640, G9141, and G9142.

${ }^{9}$ For a discussion of the underlying assumptions of this model and their implications, please see Finkelstein et al. (2016).
} 
to outcome $y$ ) on patient $i$ 's outcome, where $d(i, t)$ indexes the physician attributed to patient $i$ in year t. $x_{i t}$ is a vector of time-varying patient characteristic ${ }^{10}$ and calendar year fixed effects.

Our goal is to estimate the degree to which variation in patient utilization across physicians is attributable to the physicians' practice styles, thus capturing the impact that PCPs have on patient care. This requires a plausibly exogenous change in the practice styles of a patient's physician. Specifically, we need to utilize a source of variation that is presumably uncorrelated with the patient's underlying evolution in health care utilization in the absence of the change. To achieve this goal, we analyze outcomes of patients who experienced a change in their assigned primary care physician, where the change is triggered by their original physician's geographical relocation or exit from the Medicare program (collectively referred to as "exits"). We use the difference in practice styles between the patient's original and new physician as the source of variation. We discuss the identifying assumption and its validity below.

For patient $i$, whose assigned physician changed from $d_{0}$ to $d_{1}$, due to $d_{0}$ 's exit, we denote $\Delta_{i}$ as the difference in average utilization between the new and the original physician: $\Delta_{i} \equiv y_{d_{1}}-y_{d_{0}}$, where $y_{d} \equiv E\left[y_{i d t}\right]$ is the average taken over all time periods and all patients assigned to physician $d$. Empirically, we include in these averages all patients besides patient $i$ and use "leave-one-out" means. Prior to calculating this difference, we apply Bayesian shrinkage to reduce the measurement error in the physician means since some PCPs have few assigned patients. The empirical Bayes method scales each PCP's estimated mean by a function of its variance to shrink the mean toward the overall average across all PCPs. ${ }^{11}$ To summarize, $\Delta_{i}$ is the sum of the differences in the physicians' and patients' contributions to observed health care utilization differences across physicians' patients..$^{12}$ Note that we do not risk-adjust $\Delta_{i}$ using patient observables because we want to retain a specific interpretation of our estimates, and we discuss that interpretation below.

Next, we denote the difference across physicians $d_{0}$ and $d_{1}$ that is attributable to physician practice styles as: $\theta \equiv \frac{\delta_{d_{1}}-\delta_{d_{0}}}{y_{d_{1}}-y_{d_{0}}}$. We define $r(i, t)$ as the period relative to the physician's exit, and

\footnotetext{
${ }^{10}$ Specifically, we include a quadratic in age and indicators for Medicare coverage characteristics listed in the second panel of Table 1 .

${ }^{11}$ We have estimated our results without using empirical Bayes shrinkage and find very similar, albeit noisier, results.

${ }^{12}$ Note that in our application "place effects" are absorbed by the "patient" component.
} 
we let $I_{r(i, t) \geq 0}$ denote an indicator for the exit event. For patients whose physicians exit, we can write:

$$
y_{i t}=\alpha_{i}+\delta_{d_{0}}+\theta I_{r(i, t) \geq 0} \Delta_{i}+x_{i t} \beta+\varepsilon_{i t},
$$

where $\theta$ is our parameter of interest. It represents the average change in patients' health care utilization in the years following physician exits, relative to the overall difference between the new and old physicians' practice style means; in short, under the identifying assumption, it captures the variation across PCPs that can be explained by the PCPs' practice styles.

This intuitive interpretation of $\theta$, as a share of the overall variation across PCPs, relies on the way we define $\Delta_{i}$; specifically, the fact that we include all of the variation across physicians without risk adjustments. In the hypothetical case of perfect risk adjustment (i.e., where all of the variation is purged of patient-specific factors including health and preferences), $\theta$ would converge to 1 by its definition. Consistent with this observation, we find larger estimates for $\theta$ when we risk adjust using observable patient characteristics (through the $x_{i t} \beta$ component). ${ }^{13}$ We take advantage of this feature later in the analysis.

\subsection{Estimating Equations}

Based on the latter equation, we take two specifications to the data. The first specification is the direct empirical analogue for estimating the mean effects in the post-exit years, which takes a standard difference-in-differences form:

$$
y_{i t}=\mu_{i}+\theta \times \text { Post }_{i t} \times \Delta_{i}+x_{i t} \beta+\varepsilon_{i t},
$$

where $\mu_{i}=\alpha_{i}+\delta_{d_{0}}$. Here, Post it $_{\text {it }}$ is andicator variable that equals 1 in the post-exit years and equals 0 in the pre-exit years, and the vector $x_{i t}$ also includes the variable Post $t_{i t}{ }^{14}$ For inference,

\footnotetext{
${ }^{13}$ For example, the estimate for office visits increases from 0.54 to 0.69 and the estimate for physician spending increases from 0.54 to 0.74 when we risk-adjust using all of the patient characteristics in Table 1.

${ }^{14}$ Note that $\Delta_{i}$ is absorbed by the patient fixed effect.
} 
we take a relatively conservative approach and estimate robust standard errors clustered at the HRR-level.

Our second specification allows for flexible interactions with time with respect to the event. Specifically, we estimate the following corresponding event-study equation:

$$
y_{i t}=\mu_{i}+\sum_{r \neq-1, r=-4}^{r=6} \theta_{r} \times I_{r} \times \Delta_{i}+x_{i t} \beta+\varepsilon_{i t}
$$

where $I_{r}=1(r(i, t)=r)$. In this specification, our baseline period is the year prior to the exit $(r=-1)$, and the indicators for time relative to the exit $\left(I_{r}\right.$ for $\left.r \neq-1\right)$ are included in the vector $x_{i t}$. The event study specification allows us to test for parallel trends in the pre-period (based on $\theta_{r}$ for $r<-1$ ), as well as to investigate potential dynamics in the post-period (based on $\theta_{r}$ for $r>0$ ).

Finally, toward the end of our empirical analysis, we extend our main investigation to analyzing average "cross-outcome" effects, to study the degree of complementarity across different types of health care utilization. That is, we show how variation in PCP intensity in dimension $m$ (specifically, office-based care) correlates with variation in patient health care utilization dimension $n$ (e.g., inpatient spending). Unlike in our main analysis, however, we now risk-adjust $\Delta_{i}$ with all of the patient characteristics and chronic conditions contained in the data (listed in Table 1). We risk-adjust the change in practice styles here in order to have a larger "first stage"; we want the outcome-on-outcome effect of office-based care to be closer to one to ease the interpretation of the cross-outcome ("reduced form") effects. This approach differs from our main analysis where we focus on estimating the share of utilization that is attributable to PCPs. Our cross-outcome difference-in-differences estimating equation takes the form:

$$
y_{i t}^{n}=\mu_{i}^{n}+\theta \times \text { Post }_{i t} \times \Delta_{i}^{m}+x_{i t} \beta^{n}+\varepsilon_{i t}^{n},
$$

where $\mu_{i}^{n}=\alpha_{i}^{n}+\delta_{d_{0}}^{n}$ and $\theta \equiv \frac{\delta_{d_{1}}^{n}-\delta_{d_{0}}^{n}}{\tilde{y}_{d_{1}}^{m}-\tilde{y}_{d_{0}}^{m}}$ (with $\tilde{y}_{d}^{m} \equiv E\left[y_{i d t}^{m}-x_{i t} \beta^{m}\right]$ ); so $\theta$ now measures the rate at which differences in office-based practice style $m$ translate to changes in utilization outcome $n$. 
We measure office-based practice styles using physician spending on office-based care per patient and the number of office visits per patient.

\subsection{Identifying Assumption and Validity}

Our identifying assumption is that patients who get exposed to different changes in practice styles (i.e., $\Delta_{i}$ ) following the original PCP exit have health care utilization that would run parallel in the absence of the exit. Put differently, our design requires that patients' underlying trends in health care utilization do not systematically differ by $\Delta_{i}$. Given that patient separations from their old PCPs are triggered by PCP exits, the separation side of the induced variation in practice styles is likely exogenous to patient characteristics and health care utilization trajectories. However, within the source of variation that we utilize, there could still be selection into new PCPs following the exits.

To address this concern, we provide two sets of results. First, a testable necessary condition of our identifying assumption is the absence of differential trends in the pre-exit period across patients with differential $\Delta_{i}$. Reassuringly, we show that there are virtually no differential trends across differentially treated patients in the years prior to the exit for all outcomes. This alleviates concerns that patients who switch to higher or lower intensity PCPs might be on different trajectories. Second, we use observable patient characteristics and health care conditions in the pre-exit period to try to predict each patient's change in PCP practice style intensity, $\Delta_{i}$. Based on this analysis, we find minimal evidence of selection into PCPs with differential practice style intensity, and we discuss these results in more detail in the next section.

The signature feature of our quasi-experiment is that it relies on sharp changes in utilization around the time of PCP exits across patients who experience differential changes in practice styles, $\Delta_{i}$. As such, our design cannot account for shocks to health care utilization (such as adverse health events) that both differ across patients with varying $\Delta_{i}$ and align exactly with the timing of exits. However, we believe that this concern is mitigated in our setting, because patients switch PCPs due to their original PCP exiting, rather than due to their own behavior (such as patient relocation 
or endogenous switches after, e.g., the discovery of a new medical condition) 15

\section{Results}

We now present our results showing how PCP practice styles affect patient health care utilization. The first set of results describes the variation across patients in their exposure to different practice styles following PCP exits. The second set of results shows, in an event study framework, how switching to higher intensity PCPs affects health care utilization around the date of the original PCP exit. We follow the event study results with standard difference-in-difference results that summarize the average longer-run effects of switching to higher intensity PCPs. As robustness, we examine how patient-PCP selection on observables could affect our results. Finally, we study how switching to PCPs who provide more office-based care affects utilization of other types of health care. The latter exercise reveals whether office-based care substitutes for or complements other types of health care, which could help policymakers anticipate the effects of changing reimbursement rates for office-based care (Song and Gondi 2019).

\subsection{Distributions of Changes in PCP Practice Styles Following Exits}

Recall that our identifying variation comes from the difference between the new PCP practice style and the original PCP practice style for the sample of patients affected by PCP exits. We calculate this difference for the thirteen practice style measures described in Table 2, which broadly capture health care spending, office visits and diagnoses, as well as low-quality and high-quality care. Table 3 provides the mean, standard deviation, 25 th percentile, and 75 th percentile for each of the thirteen differences, i.e., $\Delta_{i}$. We expect the changes in PCP practice styles to be centered around zero, unless patients systematically seek out higher or lower intensity PCPs following exits, and the means in column 1 confirm this hypothesis. Reading across the first row shows, for exam-

\footnotetext{
${ }^{15}$ Consistent with this view, we do not find evidence that patients systematically switch to higher intensity PCPs following their original PCPs' exits, unlike what we might expect to find if patients switched physicians in response to health shocks.
} 
ple, that the average patient switched to a PCP with \$6 lower physician spending, while the patient at the 25th percentile switched to a PCP with $\$ 117$ lower physician spending (compared to her original PCP), and the patient at the 75th percentile switched to a PCP with \$108 higher physician spending (compared to her original PCP). The variation in total spending practice styles (row 6) is

much larger; the patient at the 25th percentile switched to a PCP with $\$ 2,126$ lower total spending and the patient at the 75 th percentile switched to a PCP with $\$ 2,776$ higher total spending. Overall, Table 3 shows that patients get exposed to significant variation in the PCP practice styles following PCP exits, and this variation provides the statistical foundation for our identification strategy. The table also provides a reference for considering the magnitudes of the effects that we present in subsequent sections.

\subsection{Effects of Switching to Higher Intensity PCPs}

\subsubsection{Event Study Results}

Next, we present our core analysis of the effects of switching to higher intensity PCPs across our range of health care utilization outcomes. We begin by describing results from estimating the event study specification (equation 2). The event study estimates make two contributions: first, they reveal whether patients who switch to higher intensity PCPs exhibit differential pre-trends, which speaks to the internal validity of our quasi-experimental design, and second, they show how PCP practice style intensity dynamically affects patients' health care utilization in the post-exit years.

Figure 1 plots our event study estimates for the spending-based practice style outcomes, and Figure 2 plots our estimates for the office visits, diagnoses, "low-quality" care, and "high-quality" care outcomes. Note that due to our patient-physician assignment procedure, period 0 is a "transitional" year, and period 1 is the first year in which all patients in our sample have fully switched to the new PCP. Therefore, we focus on period 1 as the first period that captures the "full" impact of the switch. Examining these figures reveals, in support of our research design, that there are no systematic pre-trends across patients who experience differential degrees of changes in PCP inten- 
sity. In the few cases where the pre-trends are statistically significant (given the large sample size), they are economically negligible. Meanwhile, we find that PCP practice styles have immediately large and persistent effects on patient health care utilization for all outcomes that we examine.

We find that PCP practice styles explain more of the variation in utilization across PCPs when that utilization is more closely tied to the PCP's "locus of control," as manifested in the comparison of physician spending (Figure 1.A) and total spending (Figure 1.F). When patients switch to PCPs with $\$ 1$ higher physician spending per patient, their physician spending increases by 57 cents in the first year after the PCP exit, and then stabilizes at 54 cents higher thereafter. By comparison, when patients switch to PCPs with $\$ 1$ higher total spending per patient, their total spending increases by 48 cents in the year of the exit, but then decreases to 31 cents higher spending in the post-switch years, indicating that PCPs may have less control over total spending in the long run. Overall Figure 1 shows that PCP practice styles explain about 40-55\% of the variation in physician spending and outpatient spending across PCPs, but only about $30-40 \%$ of the variation in pharmaceutical drug spending, inpatient spending, post-acute care spending, and total spending.

Figure 2 shows similar results for non-spending utilization outcomes. For example, when patients switch to PCPs whose patients have one more office visit per year, their own number of office visits increases by about 0.57 (Figure 2.A). Figures 2.B and 2.C show how patients are affected by their PCPs' propensities to diagnose medical conditions. Patients who switch to PCPs whose patients have one more unique ICD-9 diagnosis code on their records see their own number of diagnoses increase by 0.5 , and patients who switch to PCPs whose patients have one more chronic condition on their records see their own number of diagnosed chronic conditions increase by 0.4. The fact that primary care physician assignments have such large effects on the number of diagnoses that patients receive may be somewhat surprising at first, but is consistent with previous literature showing that diagnostic intensity varies across providers (Song et al. 2010; Gowrisankaran et al. 2017). Our results provide new evidence on the causal effects of PCP practice styles in explaining variation in medical diagnoses across patients. Our results also suggest that PCPs have the potential to affect patient health in the long run, since we show that PCPs determine, in part, 
which medical conditions get diagnosed and treated.

To more directly examine the potential effects of PCP practice styles on patient health, we evaluate practice styles associated with low-quality and high-quality care. As described in the data section, we use emergency department visits and avoidable hospitalizations to measure lowquality care. We find that the number of emergency department visits increases by 0.3 for patients who switch to PCPs whose patients have, on average, one more emergency department visit per year (Figure 2.D). We also find that the number of avoidable hospitalizations increases by approximately 0.5 for patients who switch to PCPs whose patients have one more avoidable hospitalization per year (Figure 2.E). On the opposite side, for high-quality care, we focus on practice styles related to influenza immunization and the provision of guideline-consistent care for diabetic patients. We find that the probability of flu vaccination increases by 6 percentage points for patients who switch to PCPs whose influenza vaccination rates are 10 percentage points higher (Figure 2.F). Even more, we find that the probability of receiving guideline-consistent care increases by 8.1 percentage points for diabetic patients (as defined in the pre-event period) who switch to PCPs whose guideline-consistent diabetic care rates are 10 percentage points higher (Figure 2.G). These results provide strong evidence that PCPs are significantly responsible for variation in patients' receipt of high- and low-quality primary care.

\subsubsection{Difference-in-Differences Results}

To summarize our event study results, we estimate a standard difference-in-differences model using equation (1), where we interact each patient's change in PCP practice style intensity with the post-exit indicator, and we estimate this model for all thirteen practice style measures. To summarize the average long-run effects, we exclude period 0 (the transitional year) and, guided by the event study results, we also exclude the first year following the event (period 1). This is because the PCP effects tend to be larger on-impact when the match is first established and they

tend to stabilize thereafter (see Figures 1 and 2). Hence, years 2 to 6 are included in the post-event periods and years -4 to -1 are included in the pre-event periods. 
The difference-in-differences results are provided in Table 4. As before, PCP practice styles have the largest effects on physician spending, office visits, and the receipt of guideline-consistent care, but they also have large effects on all other measures of utilization. The share of variation in utilization that is attributable to PCP practice styles ranges from 0.81 for recommended diabetes care to 0.26 for the number of emergency department visits and post-acute care spending. To put the magnitudes into perspective, physician spending increases by $\$ 115(=\$ 213 * 0.54)$ for patients who switch to PCPs with 1-standard deviation higher physician spending practice styles compared to the exiting PCPs. To give another example, the total number of unique diagnoses increases by $1.36(=3.31 * 0.41)$ for patients who switch to PCPs whose patients have on average 1-standard deviation more diagnoses than the patients of the exiting PCPs. Similar calculations reveal that PCP practice styles have economically meaningful effects on all forms of utilization that we measure.

In both our event study and difference-in-differences results, we assume linearity in the effect of PCP practice styles on patients' health care utilization. However, it is possible for example, that switching to higher intensity PCPs has different effects on utilization than switching to lower intensity PCPs, particularly if high utilization is an absorbing state. To investigate the linearity assumption, we re-estimate the difference-in-differences results for patients who switch to PCPs with higher vs. lower physician spending. Instead of interacting the change in the physician spending practice style with the post-event period, which imposes linearity, we now split the change in the physician spending practice style $\left(\Delta_{i}\right)$ into deciles and interact decile indicators with the post-event period indicator. We include the post-event indicator in the regression and choose the omitted category to be the decile centered on zero, where patients experience very little change in physician spending practice styles (the fifth decile). We display the estimates to scale in Figure 3; i.e., we plot the difference-in-differences estimate for a given decile against the mean change in physician spending $\left(\Delta_{i}\right)$ within that decile. The results show a surprisingly linear relationship between changes in PCP practice style intensity and changes in physician spending. Therefore, we conclude that the effects of PCP practice styles are relatively symmetric for patients experiencing increases 
or decreases in PCP intensities 16

\subsection{The Nature of Patient-Physician Re-Assignment}

Using PCP exits, our estimates from the previous section suggest that PCP practice styles have substantial and long-lasting effects on patients' health care utilization. A natural question, related to the source of variation that we use, is how patients select new physicians after their original PCPs exit. We explore that question now.

First, using provider tax identification numbers, we find that $46 \%$ of patients switch to PCPs who belong to the same group practices as their exiting PCPs and that the remaining $54 \%$ of patients switch to PCPs belonging to different practices. Figure 4 further shows how changes in the physician spending practice style vary across patients who switched to PCPs belonging to the same vs. different practices. The figure shows that patients who switch to PCPs belonging to the same practice have smaller changes in their practice style exposure compared to patients who switch to PCPs belonging to different practices. Thus, more of our identifying variation comes from the latter group, though each group provides about the same share of patients who experience increases or decreases in their practice style exposure, given that both distributions are approximately centered around zero.

Second, we assess the degree to which our estimated treatment effects could be explained by potential selection into the new patient-PCP match based on observables. To combine all the information available in our data in a systematic way, we proceed as follows. For each utilization outcome $y_{i t}$, we begin by creating a prediction as a function of pre-exit patient characteristics, using all of the demographic variables and indicators for chronic and disabling conditions contained in the MBSF (which are listed in Table 1) ${ }^{17}$ Using these predictions, we then estimate regressions to test whether patients with higher predicted utilization for outcome $y$ are more likely to switch to PCPs with higher intensity practice styles for outcome $y$. We control for HRR fixed effects and

\footnotetext{
${ }^{16}$ We find similar linearity results for the other practice style measures, which are available on request.

${ }^{17} \mathrm{We}$ do not include the chronic and disabling conditions when predicting the number of diagnoses or the number of chronic conditions, for which we only include patient characteristics from the top two panels of Table 1.
} 
year fixed effects, and bootstrap the standard errors. We perform this procedure for all thirteen changes in practice styles and present the results in Table 5.

Perhaps not surprisingly, given the large sample size and the large number of patient characteristics that we use, we find statistically significant predictability. However, the magnitudes of the coefficients are small and there is no systematic pattern. For example, in 8 cases the coefficients have a positive sign and in 5 cases the coefficients have a negative sign. By comparison, all of our estimated treatment effects were systematically positive (and large). Moreover, all of the estimates in Table 5 are small because they represent the association between a one-unit increase in the predicted outcome $y$ and the corresponding change in the PCP practice style for that outcome. As an example, we find that patients with $\$ 1$ higher predicted total spending switch to PCPs with total spending practice styles that are only $\$ 0.02$ higher. To gauge the magnitudes in a complementary way, we estimated the same set of models after standardizing both the predicted patient utilization and the changes in PCP practice styles, and the magnitudes were similarly small. For example, we found that patients with 1-standard deviation higher predicted total spending switch to PCPs with only 0.03-standard deviations higher spending relative to their original PCPs. Overall, the findings in Table 5 suggest, with a high degree of precision, that sorting on observables is relatively inconsequential in relation to the magnitudes of our main results. We think these results show there is minimal selection on observables in our design, which is also supported by the lack of systematic pre-trends in the event studies.

\subsection{Correlations Across Practice Style Measures}

There is some debate among policymakers as to whether primary care is a substitute for or a complement to other forms of health care utilization. Early research showed that people with less access to primary care were more likely to become hospitalized for ambulatory care sensitive conditions (Bindman et al. 1995), suggesting that primary care visits and hospitalizations were somewhat substitutable. More recent research, however, suggests that primary care complements other forms of health care utilization. Baicker et al. (2013) and Taubman et al. (2014) show that 
people with better access to primary care through Medicaid are more likely to receive preventive care services and they are more likely to visit emergency departments. Similarly, Cabral and Cullen (2017) show that preventive care complements non-preventive care in a sample of patients with private health insurance. Since preventive care is often provided in primary care physicians' offices, extending the results from Cabral and Cullen (2017) to our own setting could suggest complementarities between primary care and other forms of utilization. To test this hypothesis directly, we estimate equation (3) to show how switching to PCPs who provide more office-based care, as measured by practice styles that involve more office visits and physician spending, affects other types of health care utilization.

Tables 6 and 7 show the degree to which the quantity of office-based care complements or substitutes for other types of patient care 18 In Table 6, we find that total spending increases by $\$ 299$ for patients who switch to PCPs whose patients have \$100 higher physician spending per year (risk-adjusted). Of the $\$ 299$ increase, $\$ 74$ is spent on physician services, $\$ 93$ is spent on outpatient care, and $\$ 55$ is spent on pharmaceutical drugs. The relationship between office-based spending and inpatient and post-acute care spending is not statistically significant. Patients have 0.82 more office visits, but no changes in the number of emergency department visits or avoidable hospitalizations. They have 0.56 more ICD-9 diagnoses on their records, but only 0.04 more chronic conditions, suggesting that the extra office-based care might help refine existing diagnoses and/or treat existing chronic conditions, rather than help diagnose new chronic conditions. Lastly, patients whose PCPs provide more office-based care are 1.2 percentage points $(2.6 \%)$ more likely to receive flu vaccinations, and diabetic patients are 1.1 percentage points $(2.4 \%)$ more likely to receive guideline-consistent diabetes care. In Table 7, we use the number of office visits to measure the intensity of office-based care, and generally find similar results. Thus, office-based care seems to complement some types of patient care (such as outpatient spending), but does not seem to impact potentially avoidable high-cost utilization (such as the number of avoidable hospitalizations

\footnotetext{
${ }^{18}$ In these tables we report the "reduced-form" estimates of switching to PCPs whose patients receive one more unit of office-based care. To help the reader scale the correlation between a patient's own office-based care and other forms of utilization, we also report the "first-stage" estimates for the effects on a patient's own in-office care. It is 0.74 for physician spending (in Table 6) and 0.69 for office visits (in Table 7).
} 
and emergency department visits).

\section{Conclusion}

In this paper, we use several dimensions of health care utilization to characterize primary care physician practice styles, ranging from physician spending to diagnostic intensity and vaccination rates, to estimate how much of the variation in utilization across PCPs is attributable to differences in their behavior. We estimate the short-run and longer-run effects of switching to PCPs with more "intensive" practice styles by using a quasi-experiment that leverages PCP relocations and retirements to generate variation in practice style intensity within patients over time. We find that PCP practice styles affect all types of utilization that we analyze, which include measures of both quantity and quality of care. PCPs typically have the largest effects on patient utilization in the first year of a new patient match, but their strong influence persists throughout our analysis window for up to 6 years thereafter. Intuitively, PCP practice styles explain more of the variation in health care services that are more frequently provided in primary care settings, such as office-based care, the numbers of diagnoses that patients receive, influenza vaccination rates, and guideline-consistent care for diabetic patients. Nevertheless, PCP practice styles can also explain a significant share of the variation in more "distant" types of utilization, such as inpatient spending and emergency department visits. Thus, we conclude that the practice styles of primary care physicians could have long-lasting and far-reaching consequences for patient health.

Given the importance of PCP practice styles for patient health care utilization trajectories, policies that target the beliefs and behaviors of PCPs may have considerable impacts. For example, the Center for Medicare and Medicaid Services (CMS) could develop continuing education modules to raise awareness among PCPs about high-quality vs. low-quality care, and it could also change reimbursement rates to incentivize the provision of high-quality care. Since we show that PCP practice styles explain a large share of the variation in high-quality primary care that patients re-

ceive, encouraging PCPs to provide such guidelines-consistent care to all relevant patients might 
be particularly effective. In line with our analysis, CMS could additionally measure PCP practice styles related to quality of care and release that information to both physicians and their patients. This might benefit PCPs through learning how their peers treat patients in similar contexts, and it might benefit patients through their ability to choose higher-quality PCPs. As such, publishing information about physician practice styles might reduce unwarranted variation in utilization across patients, and in turn, improve the quality of care that patients receive. 


\section{References}

[1] Agha, Leila, Keith Ericson, Kimberly Geissler, and James Rebitzer. 2018. "Team Formation and Performance: Evidence from Healthcare Referral Networks." NBER Working Paper No. 24339.

[2] Alkalay, Adi, Alon Eizenberg, Amnon Lahad, and Ity Shurtz. 2019. "Physician Workload and Treatment Choice: The Case of Primary Care." CEPR Discussion Paper 13157.

[3] Baicker, Katherine, Sarah Taubman, Heidi Allen, Mira Bernstein, Jonathan Gruber, Joseph Newhouse, Eric Schneider, Bill Wright, Alan Zaslavsky, and Amy Finkelstein. 2013. “The Oregon Experiment - Effects of Medicaid on Clinical Outcomes.” New England Journal of Medicine 368: 1713-1722.

[4] Bailey, Martha J., and Andrew Goodman-Bacon. 2015. "The War on Poverty's Experiment in Public Medicine: Community Health Centers and the Mortality of Older Americans." American Economic Review, 105 (3): 1067-1104.

[5] Bindman, AB, K Grumbach, D Osmond, M Komaromy, K Vranizan, N Lurie, J Billings, and A Stewart. 1995. "Preventable Hospitalizations and Access to Care." Journal of the American Medical Association 274 (4): 305-311.

[6] Cabral, Marika, and Mark R. Cullen. 2017. "The Effect of Insurance Coverage on Preventive Care.” Economic Inquiry 55 (3): 1452-1467.

[7] Centers for Disease Control and Prevention. 2019a. "Recommended Adult Immunization Schedule."

[8] Centers for Disease Control and Prevention. 2019b. "People 65 Years and Older and Influenza." 
[9] Centers for Medicare and Medicaid Services. 2017. "2015 Measure Information about the Hospital Admissions for Acute and Chronic Ambulatory Care Sensitive Condition (ACSC) Composite Measures, Calculated For the 2017 Value-Based Payment Modifier Program."

[10] Centers for Medicare and Medicaid Services. 2019. "National Health Expenditure Projections 2018-2027."

[11] Chan, David. 2016. "Teamwork and Moral Hazard: Evidence from the Emergency Department." Journal of Political Economy, 124 (3): 734-770.

[12] Currie, Janet, and W. Bentley MacLeod. 2016. "Diagnosing Expertise: Human Capital, Decision Making, and Performance Among Physicians.” Journal of Labor Economics, 35 (1): $1-43$.

[13] Currie, Janet, W. Bentley MacLeod, and Jessica Van Parys. 2016. "Provider Practice Style and Patient Health Outcomes: The Case of Heart Attacks." Journal of Health Economics, 47: 64-80.

[14] Cutler, David M., and Dan P. Ly. 2011. "The (Paper) Work of Medicine: Understanding International Medical Costs." Journal of Economic Perspectives, 25 (2): 3-25.

[15] Cutler, David M., Jonathan S. Skinner, Ariel Dora Stern, and David Wennberg. 2019. "Physician Beliefs and Patient Preferences: A New Look at Regional Variation in Health Care Spending." American Economic Journal: Economic Policy, 11 (1): 192-221.

[16] Doyle, Joseph J. 2011. "Returns to Local-Area Health Care Spending: Evidence from Health Shocks to Patients Far from Home." American Economic Journal: Applied Economics, 3 (3): 221-43.

[17] Doyle, Joseph J., John A. Graves, and Jonathan Gruber. Forthcoming. "Evaluating Measures of Hospital Quality: Evidence from Ambulance Referral Patterns.” Review of Economics and Statistics. 
[18] Doyle, Joseph J., John A. Graves, Jonathan Gruber, and Samuel Kleiner. 2015. "Measuring Returns to Hospital Care: Evidence from Ambulance Referral Patterns.” Journal of Political Economy, 123 (1): 170-214.

[19] Epstein, Andrew, and Sean Nicholson. 2009. "The Formation and Evolution of Physician Treatment Styles: An Application to Cesarean Sections." Journal of Health Economics, 28: $1126-1140$.

[20] Epstein, Andrew, Sean Nicholson, and David A. Asch. 2016. "The Production of and Market for New Physicians' Skill.” American Journal of Health Economics, 2 (1): 41-65.

[21] Finkelstein, Amy, Matthew Gentzkow, and Heidi Williams. 2016. "Sources of Geographic Variation in Health Care: Evidence from Patient Migration." Quarterly Journal of Economics, 131 (4): 1681-1726.

[22] Fisher, Elliott S., David E. Wennberg, Therese A. Stukel, Daniel J. Gottlieb, F. L. Lucas, and Etoile L. Pinder. 2003a. "The Implications of Regional Variations in Medicare Spending: the Content, Quality, and Accessibility of Care. Part 1.” Annals of Internal Medicine, 138 (4): 273-287.

[23] Fisher, Elliott S., David E. Wennberg, Therese A. Stukel, Daniel J. Gottlieb, F. L. Lucas, and Etoile L. Pinder. 2003b. "The Implications of Regional Variations in Medicare Spending: the Content, Quality, and Accessibility of Care. Part 2." Annals of Internal Medicine, 138 (4): 288-298.

[24] Fletcher, Jason, Leora Horwitz, and Elizabeth Bradley. 2014. "Estimating the Value Added of Attending Physicians on Patient Outcomes." NBER Working Paper No. 20534.

[25] Garber, Alan M., and Jonathan S. Skinner. 2008. "Is American Health Care Uniquely Inefficient?" Journal of Economic Perspectives, 22 (4): 27-50. 
[26] Goodman, David C., Shannon Brownlee, Chiang-Hua Chang, and Elliott S. Fisher. 2010. "Regional and Racial Variation in Primary Care and the Quality of Care among Medicare Beneficiaries." Dartmouth Atlas of Health Care.

[27] Gowrisankaran, Gautam, Keith Joiner, and Pierre-Thomas Leger. 2017. "Physician Practice Style and Healthcare Costs: Evidence from Emergency Departments." NBER Working Paper No. 24155 .

[28] Gruber, Jonathan, Thomas P. Hoe, and George Stoye. 2018. "Saving Lives by Tying Hands: The Unexpected Effects of Constraining Health Care Providers.” NBER Working Paper No. 24445.

[29] Grytten, Jostein, and Rune Sorensen. 2003. "Practice Variation and Physician-Specific Effects." Journal of Health Economics, 22 (3): 403-418.

[30] Hull, Peter. 2018. “Estimating Hospital Quality with Quasi-experimental Data.” Working Paper.

[31] Koulayev, Sergei, Emilia Simeonova, and Niels Skipper. 2017. "Can Physicians Affect Patient Adherence with Medication?" Health Economics, 26 (6): 779-794.

[32] Kwok, Jennifer. 2019. "How Do Primary Care Physicians Influence Healthcare? Evidence on Practice Styles and Switching Costs from Medicare.” Working Paper.

[33] Laird, Jessica, and Torben H. Nielsen. 2018. "The Effects of Physician Prescribing Behavior on Prescription Drug Use and Labor Supply: Evidence from Movers in Denmark.” Working Paper.

[34] Laugesen, Miriam J., and Sherry A. Glied. 2011. "Higher Fees Paid to US Physicians Drive Higher Spending for Physician Services Compared to Other Countries." Health Affair, 30(9): 1647-1656. 
[35] Molitor, David. 2018. "The Evolution of Physician Practice Styles: Evidence from Cardiologist Migration.” American Economic Journal: Economic Policy, 10 (1): 326-356.

[36] Papanicolas, Irene, Liana R. Woskie, and Ashish K. Jha. 2018. "Health Care Spending in the United States and Other High-Income Countries.” JAMA, 319(10): 1024-1039.

[37] Reddy, Ashok, Craig E. Pollack, David A. Asch, Anne Canamucio, and Rachel M. Werner. 2015. "The Effect of Primary Care Provider Turnover on Patient Experience of Care and Ambulatory Quality of Care.” JAMA Internal Medicine, 175 (7) :1157-1162.

[38] Sawyer, Bradley, and Selena Gonzales. 2017. "How Does the Quality of the US Healthcare System Compare to Other Countries." Peterson-Kaiser Health System Tracker.

[39] Schneider, Eric C., Dana O. Sarnak, David Squires, and Arnav Shah. 2017. "Mirror, Mirror 2017: International Comparison Reflects Flaws and Opportunities for Better U.S. Health Care." The Commonwealth Fund.

[40] Schneider, Eric C., and David Squires. 2017. "From Last to FirstCould the US Health Care System Become the Best in the World?" New England Journal of Medicine, 377 (10): 901903.

[41] Silver, David. 2019. "Haste or Waste? Peer Pressure and Productivity in the Emergency Department." Working Paper.

[42] Simonsen, Marianne, Lars Skipper, Niels Skipper, and Peter Rønø Thingholm. 2019. "Discontinuity in Care: Practice Closures among Primary Care Providers and Patient Health.” Working Paper.

[43] Sinaiko, Anna D., Alyna T. Chien, Michael J. Hassett, Pragya Kakani, Danielle Rodin, David J. Meyers, Belen Fraile, Meredith B. Rosenthal, and Mary Beth Landrum. 2019. "What Drives Variation in Spending for Breast Cancer Patients within Geographic Regions?" Health Services Research, 54 (1): 97-105. 
[44] Song, Yunjie, Jonathan Skinner, Julie Bynum, Jason Sutherland, John E. Wennberg, and Elliott S. Fisher. 2010. “Regional Variations in Diagnostic Practices.” New England Journal of Medicine, 363 (1): 45-53.

[45] Song, Zirui and Suhas Gondi. 2019. "Will Increasing Primary Care Spending Alone Save Money?" Journal of the American Medical Association, doi:10.1001/ jama.2019. 12016 .

[46] Taubman, Sarah, Heidi Allen, Bill Wright, Katherine Baicker, and Amy Finkelstein. 2014. "Medicaid Increases Emergency-Department Use: Evidence from Oregon Health Insurance Experiment." Science, 343 (6168): 263-268.

[47] Tu, Peter. 2017. “Quasi-experimental Evidence of Physician Effects.” Working Paper.

[48] Van Parys, Jessica. 2016. "Variation in Physician Practice Styles Within and Across Emergency Departments." PloS one, 11 (8).

[49] Zhang, Xuan. 2018. "Disruption in Primary Care and Patient Outcomes: Evidence from Physician Retirement." Working Paper. 


\section{Table 1: Summary Statistics—Patient Demographics and Health Conditions}

\begin{tabular}{|c|c|c|}
\hline Enrollee Characteristics & $\begin{array}{c}\begin{array}{c}\text { Enrollees Affected } \\
\text { by PCP Exits }\end{array} \\
\end{array}$ & $\begin{array}{c}\text { Enrollees Not } \\
\text { Affected by PCP Exits }\end{array}$ \\
\hline Female & 0.59 & 0.58 \\
\hline Age & 76.19 & 75.65 \\
\hline Black & 0.08 & 0.07 \\
\hline Hispanic & 0.02 & 0.02 \\
\hline Asian & 0.02 & 0.02 \\
\hline Other Race & 0.02 & 0.02 \\
\hline Part A coverage in months & 11.63 & 11.62 \\
\hline Part B coverage in months & 11.64 & 11.65 \\
\hline Buy-in number of months & 1.51 & 1.42 \\
\hline HMO coverage in months & 0.60 & 0.38 \\
\hline Part D coverage in months & 6.37 & 6.22 \\
\hline Part D subsidy in months & 2.40 & 2.48 \\
\hline Dual eligibility in months & 1.63 & 1.54 \\
\hline Disabled and/or ESRD status & 0.01 & 0.02 \\
\hline Alzheimer's and related dementias & 0.08 & 0.08 \\
\hline Anemia & 0.24 & 0.24 \\
\hline Atrial fibrillation & 0.09 & 0.09 \\
\hline Benign prostatic hyperplasia & 0.07 & 0.07 \\
\hline Cataracts & 0.23 & 0.23 \\
\hline Chronic kidney disease & 0.14 & 0.14 \\
\hline COPD & 0.11 & 0.12 \\
\hline Congestive heart failure & 0.15 & 0.16 \\
\hline Depression & 0.11 & 0.11 \\
\hline Diabetes & 0.27 & 0.26 \\
\hline Glaucoma & 0.12 & 0.12 \\
\hline Hyperlipidemia & 0.51 & 0.5 \\
\hline Hypertension & 0.63 & 0.61 \\
\hline Hypothyroidism & 0.10 & 0.10 \\
\hline Ischemic heart disease & 0.32 & 0.32 \\
\hline Osteoporosis & 0.08 & 0.08 \\
\hline Rheumatoid arthritis & 0.30 & 0.29 \\
\hline $\mathrm{N}=$ \#Enrollees $\mathrm{x}$ Years & $1,186,752$ & $27,402,848$ \\
\hline \# Enrollees & 195,023 & $6,346,211$ \\
\hline
\end{tabular}

Notes: This table provides sample means derived from the 20\% Medicare Master Beneficiary Summary Files and the $20 \%$ Carrier claims from the years 2007-2013. Column 1 shows information on patient characteristics, Medicare enrollment information, and chronic conditions for patients affected by PCP exits at some point during the sample period. Column 2 shows the same information for patients who were never affected by PCP exits during the sample period, but who could be attributed to a PCP using the Evaluation and Management codes in the Carrier claims. 


\section{Table 2: Summary Statistics—Patient Health Care Utilization}

\begin{tabular}{|c|c|c|}
\hline Health Care Spending & $\begin{array}{c}\text { Enrollees Affected } \\
\text { by PCP Exits }\end{array}$ & $\begin{array}{c}\text { Enrollees Not } \\
\text { Affected by PCP Exits }\end{array}$ \\
\hline Physician Spending & $\$ 615.38$ & $\$ 638.71$ \\
\hline Pharmaceutical Drug Spending & $\$ 1,877.17$ & $\$ 1,929.05$ \\
\hline Outpatient Spending & $\$ 2,551.42$ & $\$ 2,865.29$ \\
\hline Inpatient Spending & $\$ 4,654.22$ & $\$ 5,104.92$ \\
\hline Post-Acute Care Spending & $\$ 1,841.40$ & $\$ 2,016.62$ \\
\hline Total Spending & $\$ 11,634.22$ & $\$ 12,627.15$ \\
\hline \multicolumn{3}{|l|}{ Office Visits and Diagnoses } \\
\hline \# Office Visits & 8.13 & 8.61 \\
\hline \# Diagnoses & 13.36 & 14.60 \\
\hline \# Chronic Conditions & 3.74 & 3.73 \\
\hline \multicolumn{3}{|l|}{ Low-Quality Care } \\
\hline \# ED Visits & 0.54 & 0.56 \\
\hline \# Avoidable Hospitalizations & 0.05 & 0.06 \\
\hline \multicolumn{3}{|l|}{ High-Quality Care } \\
\hline $\operatorname{Pr}($ Flu Vaccine=1) & 0.45 & 0.48 \\
\hline $\operatorname{Pr}($ Recommend Diabetes Care $=1)$ & 0.46 & 0.49 \\
\hline $\mathrm{N}=$ \#Enrollees $\mathrm{x}$ Years & $1,186,752$ & $27,402,848$ \\
\hline \# Enrollees & 195,023 & $6,346,211$ \\
\hline
\end{tabular}

Notes: This table provides sample means derived from the 20\% Medicare Master Beneficiary Summary Files and the 20\% Carrier claims from the years 2007-2013. Column 1 shows information on health care utilization for patients affected by PCP exits at some point during the sample period. Column 2 shows the same information for patients who were never affected by PCP exits during the sample period, but who could be attributed to a PCP using the evaluation and management codes in the Carrier claims. 
Table 3: Distributional Moments of Changes in Practice Styles $\left(\Delta_{i}\right)$

\begin{tabular}{lcccc}
\hline \hline & Mean & Std Dev & 25th percentile & 75th percentile \\
\hline & & & & \\
Physician Spending & $-\$ 6.07$ & $\$ 212.78$ & $-\$ 117.02$ & $\$ 107.79$ \\
Pharma Drug Spending & $\$ 52.62$ & $\$ 1,181.07$ & $-\$ 414.89$ & $\$ 443.60$ \\
Outpatient Spending & $\$ 70.08$ & $\$ 1,176.46$ & $-\$ 473.84$ & $\$ 565.87$ \\
Inpatient Spending & $\$ 184.39$ & $\$ 2,699.38$ & $-\$ 1,056.25$ & $\$ 1,320.99$ \\
Post-Acute Care Spending & $\$ 97.58$ & $\$ 1,210.30$ & $-\$ 469.37$ & $\$ 638.17$ \\
Total Spending & $\$ 436.52$ & $\$ 5,419.19$ & $-\$ 2,126.40$ & $\$ 2,775.76$ \\
& & & & \\
\# Office Visits & -0.11 & 2.56 & -1.43 & 1.26 \\
\# Diagnoses & 0.11 & 3.31 & -1.78 & 1.98 \\
\# Chronic Conditions & 0.03 & 0.87 & -0.48 & 0.55 \\
\# ED Visits & 0.02 & 0.23 & -0.11 & 0.14 \\
\# Avoidable Hospitalizations & 0.003 & 0.04 & -0.02 & 0.02 \\
Pr(Flu Vaccine=1) & -0.01 & 0.16 & -0.10 & 0.09 \\
Pr(Diabetes Care=1) & 0.001 & 0.15 & -0.07 & 0.07 \\
& & & & \\
\hline \hline
\end{tabular}

Notes: This table shows the means, standard deviations, 25th percentiles, and 75th percentiles of changes in the practice style measures for patients affected by PCP exits. The mean changes in practice styles are centered around zero and the standard deviations provide information about the degree of variation the exits induce.

Table 4: Average Effects of Switching to PCPs with More Intensive Practice Styles

\begin{tabular}{|c|c|c|c|c|c|}
\hline & $\begin{array}{c}(1) \\
\text { Physician \$ } \\
\end{array}$ & $\begin{array}{c}\text { (2) } \\
\text { Pharma Drug \$ } \\
\end{array}$ & $\begin{array}{c}\text { (3) } \\
\text { Outpatient \$ } \\
\end{array}$ & $\begin{array}{c}(4) \\
\text { Inpatient \$ }\end{array}$ & $\begin{array}{c}\text { (5) } \\
\text { Post-Acute \$ } \\
\end{array}$ \\
\hline$\Delta_{\mathrm{i}} *$ Post $_{\mathrm{it}}$ & $\begin{array}{c}0.535 * * * \\
(0.0173)\end{array}$ & $\begin{array}{l}0.370 * * * \\
(0.0540)\end{array}$ & $\begin{array}{c}0.412 * * * \\
(0.0475)\end{array}$ & $\begin{array}{l}0.383 * * * \\
(0.0260)\end{array}$ & $\begin{array}{c}0.263 * * * \\
(0.0383)\end{array}$ \\
\hline & $\begin{array}{c}\text { (6) } \\
\text { Total Spending } \\
\end{array}$ & $\begin{array}{c}\text { (7) } \\
\text { \#Office Visits }\end{array}$ & $\begin{array}{c}(8) \\
\text { \#Diagnoses }\end{array}$ & $\begin{array}{c}(9) \\
\text { \#Chronic Conditions } \\
\end{array}$ & \\
\hline$\Delta_{\mathrm{i}} *$ Post $_{\mathrm{it}}$ & $\begin{array}{c}0.312 * * * \\
(0.0255)\end{array}$ & $\begin{array}{c}0.540 * * * \\
(0.0358)\end{array}$ & $\begin{array}{c}0.412 * * * \\
(0.0160)\end{array}$ & $\begin{array}{c}0.406 * * * \\
(0.0128)\end{array}$ & \\
\hline & $\begin{array}{c}\text { (10) } \\
\text { \#ED Visits }\end{array}$ & $\begin{array}{c}(11) \\
\text { \#Avoidable Hosp }\end{array}$ & $\begin{array}{c}(12) \\
\operatorname{Pr}(\text { Flu Vaccine=1) }\end{array}$ & $\begin{array}{c}(13) \\
\text { Pr(Diabetes Care=1) } \\
\end{array}$ & \\
\hline$\Delta_{\mathrm{i}} *$ Post $_{\mathrm{it}}$ & $\begin{array}{c}0.265 * * * \\
(0.0247)\end{array}$ & $\begin{array}{c}0.368 * * * \\
(0.0382)\end{array}$ & $\begin{array}{c}0.605 * * * \\
(0.0168)\end{array}$ & $\begin{array}{l}0.813 * * * \\
(0.0269)\end{array}$ & \\
\hline
\end{tabular}

Notes: This table shows difference-in-difference estimates for the average effects of switching to PCPs with more intensive practice styles. Each cell is an estimate for $\theta$ from equation (1) for a different utilization outcome $y$, which is the parameter on the interaction between the change in PCP practice style $\left(\Delta_{i}\right)$ for that outcome and an indicator for the post-event period (Post $t_{i t}$ ). To summarize the average longer-run effects, we include years 2 to 6 in the post-event periods (and we include years -4 to -1 in the pre-event periods). We control for patient fixed effects, timevarying patient characteristics, a post-event time indicator, and calendar year fixed effects. The standard errors are clustered at the HRR-level. *** $p<0.001,{ }^{* *} p<0.01,{ }^{*} p<0.05$. 


\section{Table 5: PCP-Patient Selection on Observables}

\begin{tabular}{lclc}
\hline \hline Utilization Outcome: & $\boldsymbol{\Delta}_{\mathbf{i}, y}$ & Utilization Outcome: & $\boldsymbol{\Delta}_{\mathbf{i}, \mathbf{y}}$ \\
\hline & & & \\
Predicted Physician \$ & $-0.010^{* * * *}$ & Predicted \# Diagnoses & $0.018^{* * *}$ \\
& $(0.002)$ & & $(0.003)$ \\
Predicted Pharma Drug \$ & $0.014^{* * *}$ & Predicted \# Chronic Conditions & -0.003 \\
& $(0.002)$ & & $(0.002)$ \\
Predicted Outpatient \$ & $0.016^{* * *}$ & Predicted \# ED Visits & $0.014^{* * *}$ \\
& $(0.002)$ & & $(0.001)$ \\
Predicted Inpatient \$ & $0.020^{* * *}$ & Predicted \# Avoidable Hospitalizations & $0.014^{* * *}$ \\
& $(0.002)$ & & $(0.001)$ \\
Predicted Post-Acute Care \$ & $0.014^{* * *}$ & Predicted Prob. of Flu Vaccination & $-0.010^{* * *}$ \\
& $(0.002)$ & & $(0.003)$ \\
Predicted Total \$ & $0.019^{* * *}$ & Predicted Prob. of Diabetes Care & $-0.014^{* * *}$ \\
& $(0.002)$ & & $(0.002)$ \\
Predicted \# Office Visits & $-0.016^{* * *}$ & & \\
& $(0.002)$ & & \\
\hline \hline
\end{tabular}

Notes: This table assesses the degree to which our estimated treatment effects could be explained by potential selection into the new patient-PCP match based on observables. To combine all the information available in our data in a systematic way, we proceed as follows. For each utilization outcome $y_{i t}$, we begin by creating a prediction as a function of pre-exit patient characteristics, using all of the demographic variables and indicators for chronic and disabling conditions contained in the MBSF (which are listed in Table 1). We do not include the chronic and disabling conditions when predicting the number of diagnoses or the number of chronic conditions, for which we only include patient characteristics from the top two panels of Table 1. Using these predictions, we then estimate regressions to test whether patients with higher predicted utilization for outcome $y$ are more likely to switch to PCPs with higher intensity practice styles for outcome $y$. The coefficient estimates for those associations appear in the table. We control for HRR fixed effects and year fixed effects, and we bootstrap the standard errors. *** $p<0.001, * * p<0.01, * p<0.05$. 
Table 6: Average Effects of Switching to PCPs with $\$ 100$ Higher Physician Spending Per Patient

\begin{tabular}{lclc}
\hline \hline & & \\
Utilization Outcome: & \multicolumn{3}{c}{ Utilization Outcome: } \\
\hline & $298.7 * * *$ & \# Diagnoses & $0.560^{* * *}$ \\
Total \$ & $(59.96)$ & & $(0.0383)$ \\
& $74.38^{* * *}$ & \# Chronic Conditions & $0.0359^{* * *}$ \\
Physician \$ & $(2.575)$ & & $(0.00657)$ \\
& $93.26^{* * *}$ & \# ED Visits & 0.00630 \\
Outpatient \$ & $(17.77)$ & & $(0.00341)$ \\
& $54.86^{* *}$ & \# Avoid Hospitalizations & 0.000794 \\
Pharmaceutical Drug \$ & $(17.49)$ & & $(0.000800)$ \\
& 67.93 & Prob of Flu Vaccination & $0.0117 * * *$ \\
Inpatient \$ & $(35.82)$ & & $(0.00216)$ \\
& 1.249 & Prob. of Diabetes Care & $0.0114 * * *$ \\
Post-Acute Care \$ & $(17.98)$ & & $(0.00331)$ \\
& $0.821^{* * *}$ & & \\
\# Office Visits & $(0.0597)$ & & \\
& & & \\
\hline \hline
\end{tabular}

Notes: This table shows how switching to primary care physicians with $\$ 100$ higher (risk-adjusted) physician spending per patient affects other types of health care utilization. We use the difference-in-differences specification in equation (3) to estimate these cross-outcome effects. Each cell is an estimate for $\theta$ from equation (3) for a different utilization outcome $y$, which is the parameter on the interaction between the change in PCP practice style $\left(\Delta_{i}\right)$ in physician spending and an indicator for the post-event period $\left(\right.$ Post $\left._{i t}\right)$. We include years -4 to -1 in the pre-event period, and we include years 2 to 6 in the post-event period. In these estimations, we risk-adjust $\Delta_{i}$ with all of the patient characteristics and chronic conditions contained in the data (listed in Table 1). We control for patient fixed effects, time-varying patient characteristics, a post-event time indicator, and calendar year fixed effects. The standard errors are clustered at the HRR-level. *** $p<0.001,{ }^{* *} p<0.01,{ }^{*} p<0.05$. 
Table 7: Average Effects of Switching to PCPs with One Additional Office Visit Per Patient

\begin{tabular}{lclc}
\hline \hline & & & \\
Utilization Outcome: & \multicolumn{3}{c}{ Utilization Outcome: } \\
\hline & $171.3^{* * *}$ & \# Diagnoses & $0.386^{* * *}$ \\
Total \$ & $(40.56)$ & & $(0.0252)$ \\
& $46.87^{* * *}$ & \# Chronic Conditions & 0.0114 \\
Physician \$ & $(2.368)$ & & $(0.00591)$ \\
& $56.89^{* * *}$ & \# ED Visits & 0.00178 \\
Outpatient \$ & $(15.36)$ & & $(0.00241)$ \\
& 18.31 & \# Avoid Hospitalizations & -0.000261 \\
Pharmaceutical Drug \$ & $(13.45)$ & & $(0.000621)$ \\
& $51.85^{*}$ & Prob of Flu Vaccination & $0.00758^{* * *}$ \\
Inpatient \$ & $(23.49)$ & & $(0.00180)$ \\
& -12.34 & Prob. of Diabetes Care & $0.00561 *$ \\
Post-Acute Care \$ & $(15.00)$ & & $(0.00263)$ \\
& $0.694^{* * *}$ & & \\
\# Office Visits & $(0.0496)$ & & \\
& & & \\
\hline \hline
\end{tabular}

Notes: This table shows how switching to a primary care physician with 1 more (risk-adjusted) office visit per patient affects other types of health care utilization. We use the difference-in-differences specification in equation (3) to estimate these cross-outcome effects. Each cell is an estimate for $\theta$ from equation (3) for a different utilization outcome $y$, which is the parameter on the interaction between the change in PCP practice style $\left(\Delta_{i}\right)$ in physician spending and an indicator for the post-event period $\left(\right.$ Post $\left._{i t}\right)$. We include years -4 to -1 in the pre-event period, and we include years 2 to 6 in the post-event period. In these estimations, we risk-adjust $\Delta_{i}$ with all of the patient characteristics and chronic conditions contained in the data (listed in Table 1). We control for patient fixed effects, time-varying patient characteristics, a post-event time indicator, and calendar year fixed effects. The standard errors are clustered at the HRR-level. *** $p<0.001, * * p<0.01,{ }^{*} p<0.05$. 
Figure 1: Effects of Switching to PCPs with More Intensive Spending Practice Styles $(\$ 1$ Increase)
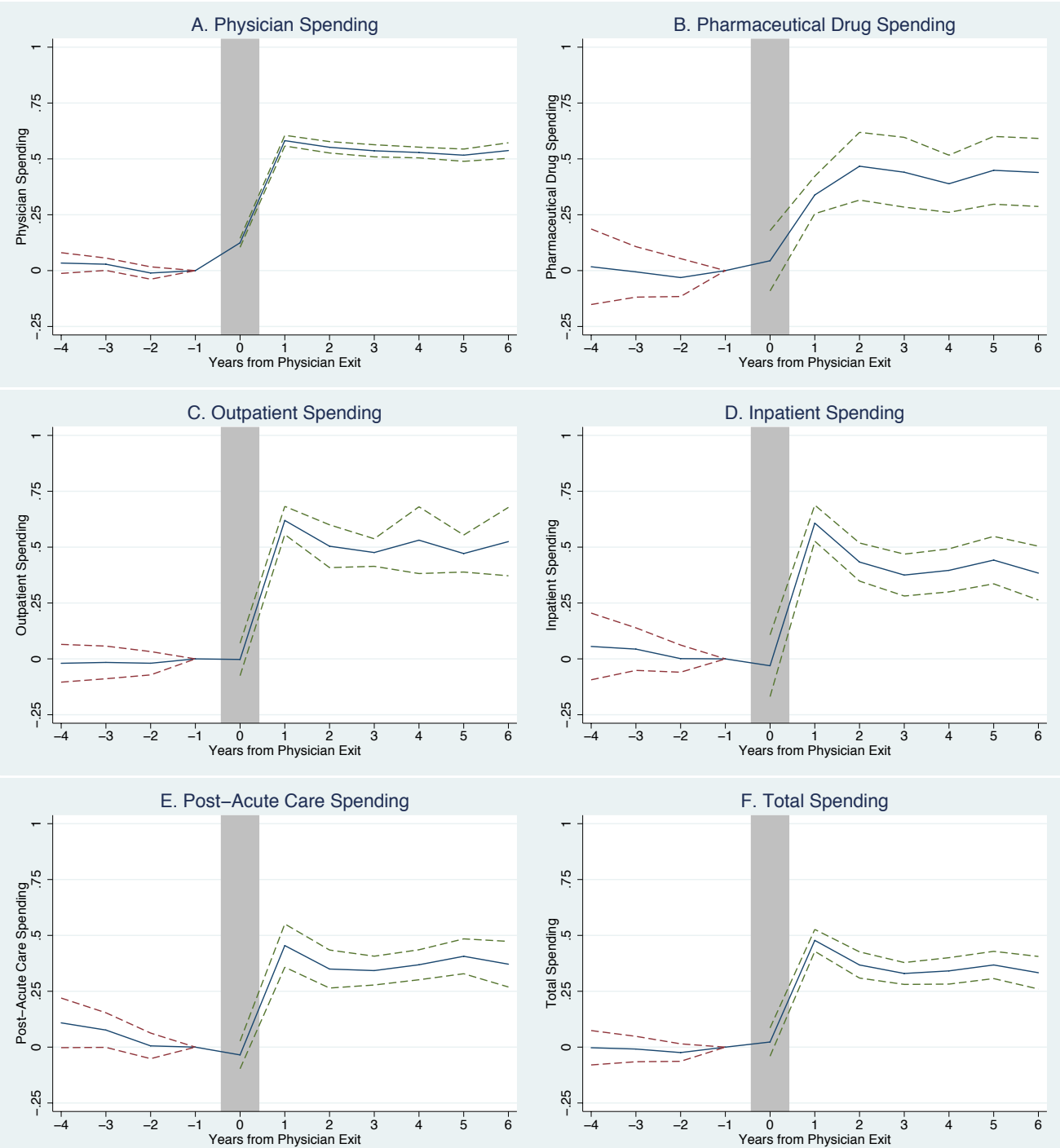

Notes: The figures show the event study estimates for patients who switch to PCPs with $\$ 1$ more intensive practice styles. Period 0 is the year in which the original PCP exits, and period 1 is the first full year after the exit in which the patient is assigned to a new PCP. Period -1 is our baseline year with respect to which all the parameters are normalized. The solid line plots estimates for the vector $\theta_{r}$ from equation (2) which captures the parameters on the interaction between the change in PCP practice styles $\left(\Delta_{i}\right)$ and indicators for time relative to the event. The dotted lines plot 95\% confidence intervals constructed from standard errors that are clustered at the HRR-level. The sample is unbalanced, though the event study estimation includes patient fixed effects, event time fixed effects, calendar year fixed effects, and time-varying patient characteristics. 
Figure 2: Effects of Switching to PCPs with More Intensive Utilization Practice Styles (1-unit Increase)
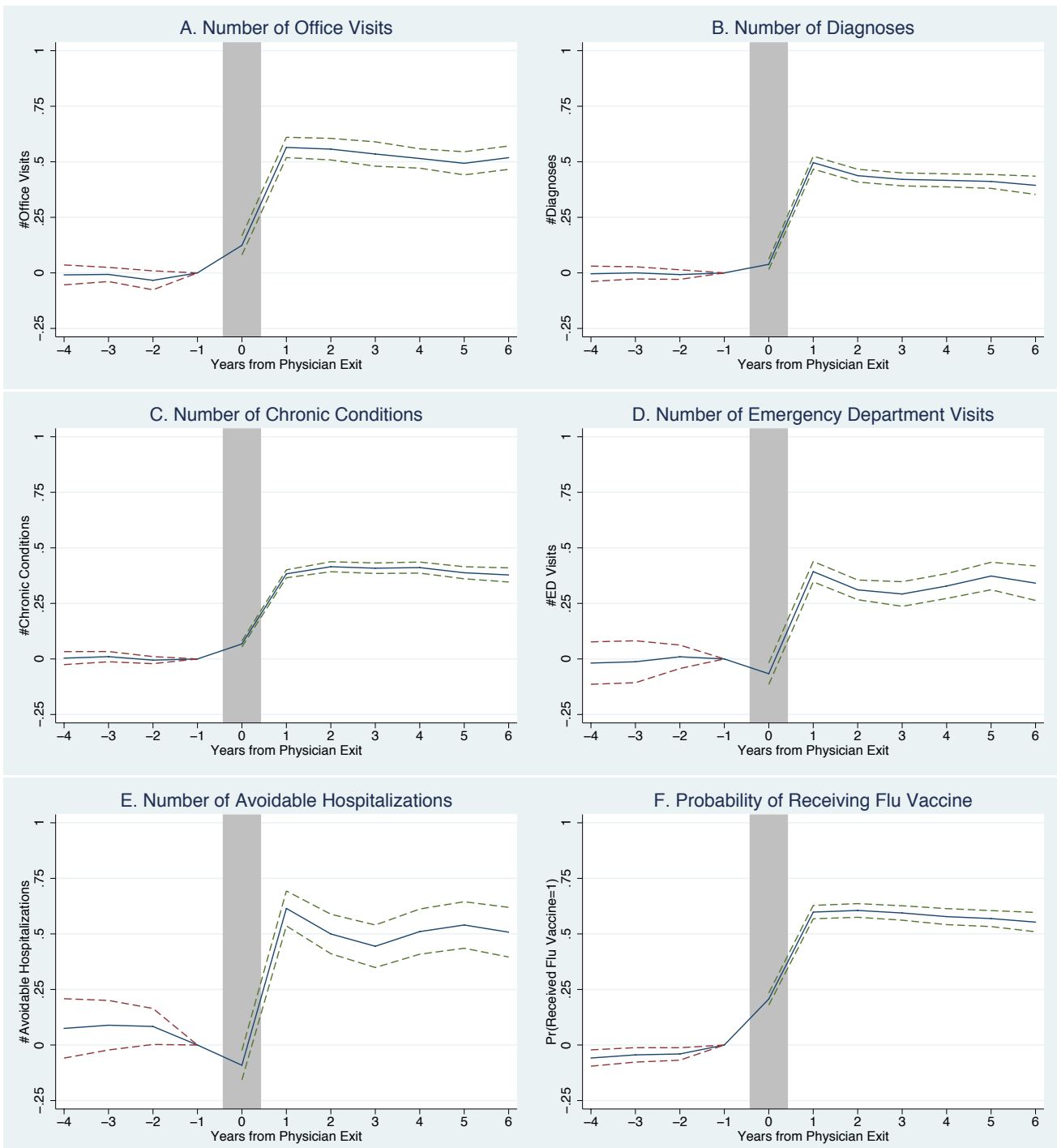

G. Probability of Receiving Diabetes Care

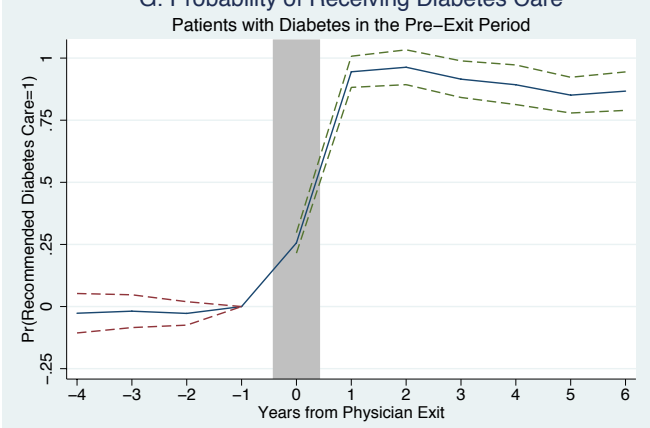

Notes: The figures show the event study estimates for patients who switch to PCPs with 1-unit more intensive practice styles. Period 0 is the year in which the original PCP exits, and period 1 is the first full year after the exit in which the patient is assigned to a new PCP. Period -1 is our baseline year with respect to which all the parameters are normalized. The solid line plots estimates for the vector $\theta_{r}$ from equation (2) which captures the parameters on the interaction between the change in PCP practice styles $\left(\Delta_{i}\right)$ and indicators for time relative to the event. The dotted lines plot 95\% confidence intervals constructed from standard errors that are clustered at the HRR-level. The sample is unbalanced, though the event study estimation includes patient fixed effects, event time fixed effects, calendar year fixed effects, and time-varying patient characteristics. 
Figure 3: Difference-in-Differences Estimates by Deciles of Changes in Physician Spending

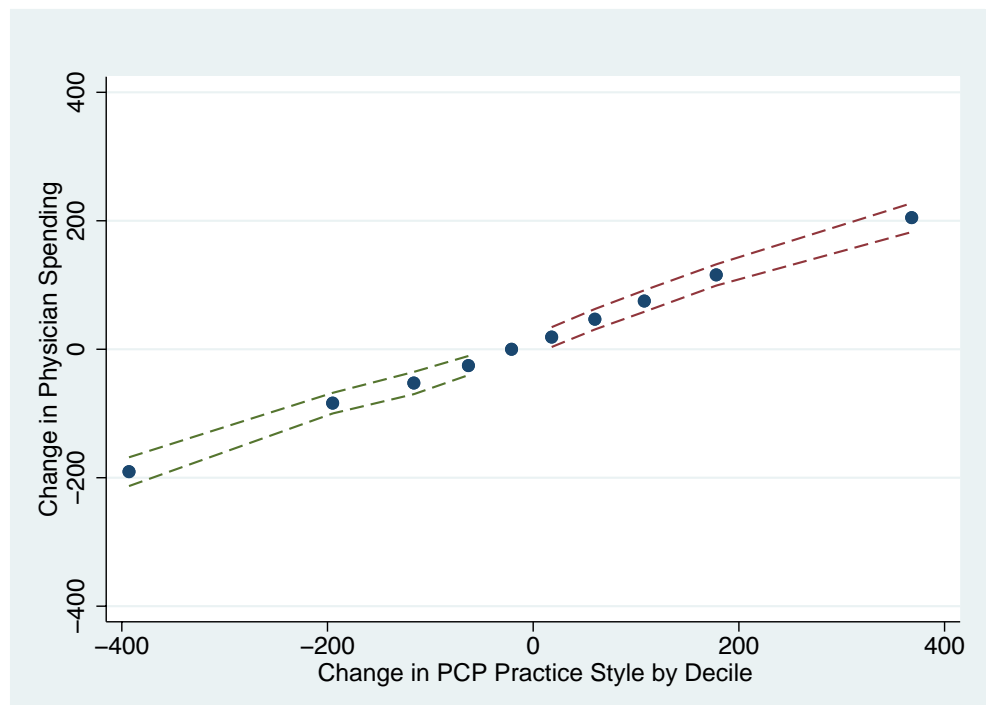

Notes: This figure plots difference-in-difference estimates for the average effects of switching PCPs by the decile of the experienced change in physicians' spending intensity. Specifically, we modify the estimation of equation (1) in the following way. We split the change in the physician spending practice style $\left(\Delta_{i}\right)$ into deciles, and we then interact decile indicators with the post-event period indicator $\left(\right.$ Post $\left._{i t}\right)$. We include years -4 to -1 in the pre-event periods, and we include years 2 to 6 in the post-event periods. The omitted category is the decile centered on zero, where patients experience very little change in physician spending practice styles (the fifth decile). We plot the mean difference-in-differences estimate for a given decile against the mean change in physician spending $\left(\Delta_{i}\right)$ within that decile. The dotted lines plot $95 \%$ confidence intervals constructed from standard errors that are clustered at the HRR- level. We control for patient fixed effects, time-varying patient characteristics, the post-event time indicator, and calendar year fixed effects.

\section{Figure 4: Distributions of Changes in Physician Spending Intensity for Patients Affected by} PCP Exits

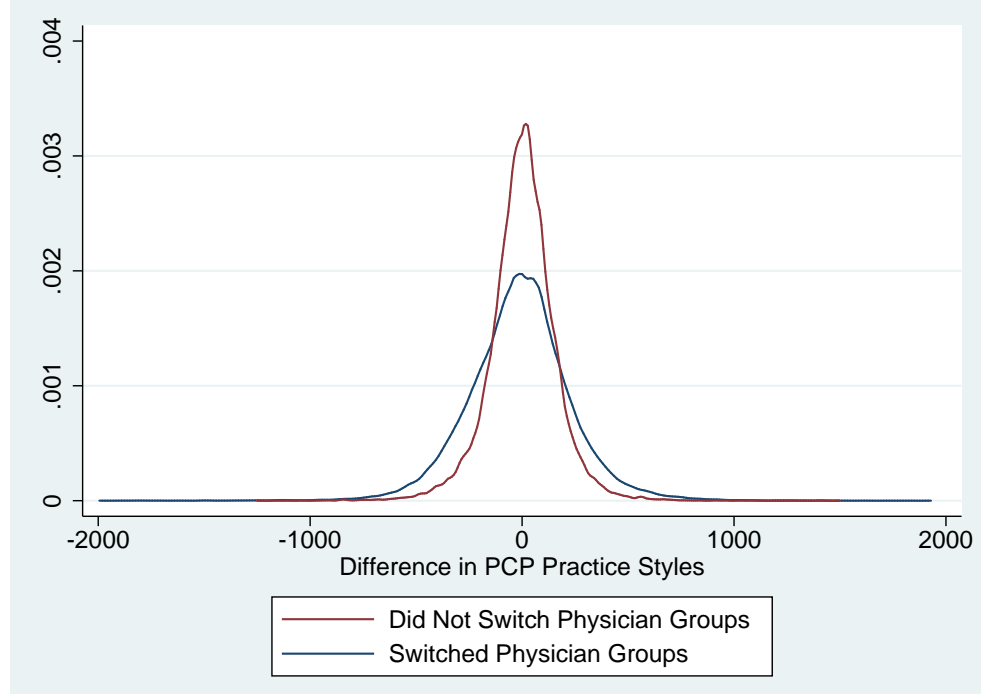

Notes: This figure plots the distributions of changes in physician spending practice styles $\left(\Delta_{i}\right)$, separately for patients who switched physician groups following PCP exits (blue distribution) and patients who remained with the same physician group following exits (red distribution). 